Supporting Information:

\title{
Oligomerisation of alkenes by radical initiation
}

\author{
Michele Cowley \\ Fischer-Tropsch Refinery Catalysis, Sasol Technology Research and Development, \\ P.O. Box 1, Sasolburg 1947, South Africa. \\ Tel: +27 16 960-3133, Fax: +27 11 522-4507,E-mail: michele.cowley@sasol.com
}

Table S1 Characterization of DTBP ${ }^{\mathrm{a}}$ by GC-FID

\begin{tabular}{ll}
\hline Component & Mass \% \\
\hline methane & 0.04 \\
ethane & 1.37 \\
1-propene & 0.01 \\
isobutane & 0.04 \\
acetone & 6.06 \\
t-butyl chloride & \\
2-me-2-propanol (t-butanol) & 0.17 \\
isobutene oxide (2,2- & 0.07 \\
dimethyl oxirane) & \\
2,2-dime butane & 0.03 \\
MEK & 0.02 \\
di-tert-butyl-peroxide & 91.03 \\
unknown & 0.00 \\
& \\
\hline TOTAL & 100.00 \\
\hline
\end{tabular}

${ }^{\mathrm{a}}$ By analysis, 99 ppm active oxygen 
Table S2 GC analysis of pentene feed and product in mass $\%$

\begin{tabular}{|c|c|c|}
\hline Component & Pentene (Feed) & Pentene (Product) \\
\hline 1-butene + isobutene & 0.2 & 0.0 \\
\hline butane & 0.3 & 0.0 \\
\hline trans-2-butene & 0.7 & 0.0 \\
\hline cis-2-butene & 0.4 & 0.0 \\
\hline 3-methyl-1-butene & 0.5 & 0.0 \\
\hline acetone & 0.6 & 0.1 \\
\hline isopentane & 3.2 & 0.7 \\
\hline 1-pentene & 5.3 & 1.5 \\
\hline 2-methyl-1-butene & 2.6 & 0.8 \\
\hline pentane & 12.7 & 6.1 \\
\hline trans-2-pentene & 45.6 & 23.4 \\
\hline 2-methyl-2-propanol + cis-2-pentene & 16.4 & 18.2 \\
\hline 2-methyl-2-butene & 10.2 & 6.3 \\
\hline 2-methyl-propanal & 0.2 & 0.1 \\
\hline 1-propanol & 0.1 & 0.2 \\
\hline cyclopentene & 0.7 & 0.5 \\
\hline cyclopentane & 0.1 & 0.1 \\
\hline 2-methylpentane & 0.1 & 0.3 \\
\hline 3-methylpentane & 0.0 & 0.2 \\
\hline methyl-ethyl ketone (MEK) & 0.1 & 0.1 \\
\hline 1-hexene & 0.0 & 0.0 \\
\hline hexane & 0.0 & 0.1 \\
\hline 2-methyl-2-pentene & 0.1 & 0.2 \\
\hline $\mathrm{C}_{6} \mathrm{H}_{12}$ & 0.0 & 0.1 \\
\hline 3-methyl-2-pentene & 0.1 & 0.2 \\
\hline 2-pentanone & 0.0 & 0.1 \\
\hline heptane & 0.0 & 0.1 \\
\hline 2,4-dimethylhexane & 0.0 & 0.1 \\
\hline 2,3-dimethylhexane & 0.0 & 0.2 \\
\hline 3-ethył2- methylpentane & 0.0 & 0.1 \\
\hline 2-methyl-heptane & 0.0 & 0.2 \\
\hline 1-octene & 0.0 & 0.9 \\
\hline m-xylene + p-xylene & 0.0 & 0.5 \\
\hline o-xylene & 0.0 & 0.2 \\
\hline $\mathrm{C}_{10}$-dimers & 0.0 & 34.2 \\
\hline $\mathrm{C}_{10} \mathrm{H}_{22}$ & 0.0 & 0.6 \\
\hline$>\mathrm{C}_{10}$ & 0.0 & 3.8 \\
\hline Total & 100.0 & 100.0 \\
\hline
\end{tabular}


Table S3 Experimental conditions and results for oligomerisation of octene and pentene with DTBP as initiator

\begin{tabular}{|c|c|c|c|c|c|c|}
\hline $\begin{array}{l}\text { Experiment } \\
\text { number }\end{array}$ & $\begin{array}{l}\text { Temperature } \\
\left({ }^{\circ} \mathrm{C}\right)\end{array}$ & $\begin{array}{l}\text { Reaction } \\
\text { Time } \\
\text { (hours) }\end{array}$ & $\begin{array}{l}\text { Initiator } \\
\text { (moles) }\end{array}$ & $\begin{array}{l}\text { Feed }^{\mathrm{c}} \\
\text { (moles) }\end{array}$ & $\begin{array}{l}\text { Benzene }^{\mathrm{h}} \\
\text { (moles) }\end{array}$ & $\begin{array}{l}\text { Products }^{\mathrm{d}} \\
(\%)\end{array}$ \\
\hline 1 & 150 & 1 & 0.00 & 0.96 & 1.68 & 0.0 \\
\hline 2 & 150 & 16 & 0.00 & 0.96 & 1.68 & 0.1 \\
\hline 3 & 150 & 1 & 0.05 & 1.85 & & 7.1 \\
\hline 4 & 150 & 1 & 0.05 & 1.85 & & 8.7 \\
\hline $5^{\mathrm{e}}$ & 150 & 1 & 0.05 & 1.85 & & 7.5 \\
\hline $6^{\mathrm{a}}$ & 150 & 3 & $0.05^{b}$ & 1.91 & & 0.0 \\
\hline $7^{\mathrm{a}}$ & 150 & 16 & $0.05^{\mathrm{b}}$ & 1.91 & & 4.8 \\
\hline 8 & 150 & 1 & $0.05^{\mathrm{b}}$ & 1.91 & & 10.4 \\
\hline 9 & 150 & 16 & $0.05^{b}$ & 1.91 & & 12.1 \\
\hline $10^{\mathrm{f}}$ & 150 & 1 & $0.08^{\mathrm{b}}$ & 1.91 & & 2.5 \\
\hline 11 & 150 & 1 & $0.08^{\mathrm{b}}$ & 1.91 & & 2.9 \\
\hline 12 & 150 & 1 & 0.05 & 0.96 & 1.68 & 2.0 \\
\hline 13 & 150 & 64 & 0.05 & 0.96 & 1.68 & 4.0 \\
\hline 14 & 150 & 2 & $0.08^{b}$ & 1.91 & & 4.5 \\
\hline 15 & 150 & 16 & $0.08^{\mathrm{b}}$ & 1.91 & & 5.3 \\
\hline 16 & 150 & 1 & 0.03 & 1.91 & & 1.3 \\
\hline 17 & 150 & 16 & 0.03 & 1.91 & & 2.1 \\
\hline 18 & 150 & 1 & 0.05 & 2.78 & & 7.1 \\
\hline 19 & 150 & 16 & 0.05 & 2.78 & & 38.0 \\
\hline 20 & 170 & 16 & 0.05 & 2.78 & & 37.2 \\
\hline 21 & 100 & 1 & 0.05 & 2.78 & & 0.4 \\
\hline 22 & 100 & 64 & 0.05 & 2.78 & & 0.9 \\
\hline 23 & 125 & 1 & 0.05 & 2.78 & & 2.7 \\
\hline 24 & 125 & 16 & 0.05 & 2.78 & & 9.9 \\
\hline 25 & 150 & 1 & 0.05 & 2.78 & & 6.4 \\
\hline 26 & 150 & 16 & 0.05 & 2.78 & & 6.6 \\
\hline 27 & 150 & 1 & 0.05 & 2.78 & & 12.2 \\
\hline 28 & 150 & 16 & 0.05 & 2.78 & & 12.2 \\
\hline 29 & 100 & 1 & 0.02 & 2.78 & & 0.2 \\
\hline 30 & 100 & 16 & 0.02 & 2.78 & & 0.2 \\
\hline 31 & 100 & 1 & 0.02 & 2.78 & & 0.2 \\
\hline 32 & 135 & 1 & 0.03 & 2.78 & & 0.7 \\
\hline 33 & 135 & 64 & 0.03 & 2.78 & & 6.5 \\
\hline 34 & 135 & 1 & 0.03 & 2.78 & & 2.8 \\
\hline 35 & 135 & 16 & 0.03 & 2.78 & & 4.8 \\
\hline 36 & 180 & 16 & 0.05 & 2.78 & & 23.2 \\
\hline 37 & 180 & 1 & 0.05 & 2.78 & & 12.7 \\
\hline 38 & 180 & 16 & 0.05 & 2.78 & & 17.7 \\
\hline $39^{\mathrm{g}}$ & 200 & 0.25 & $0.16^{\mathrm{b}}$ & 1.91 & & 12.8 \\
\hline $40^{\mathrm{g}}$ & 200 & 2 & $0.16^{\mathrm{b}}$ & 1.91 & & 15.0 \\
\hline $41^{\mathrm{g}}$ & 200 & 0.25 & $0.24^{\mathrm{b}}$ & 1.91 & & 28.1 \\
\hline $42^{\mathrm{g}}$ & 200 & 2 & $0.24^{\mathrm{b}}$ & 1.91 & & 31.4 \\
\hline $43^{g}$ & 200 & 16 & $0.24^{\mathrm{b}}$ & 1.91 & & 24.7 \\
\hline
\end{tabular}


${ }^{\mathrm{a}}$ In the presence of air. All other experiments were conducted in an argon $(99.999 \%$, Fedgas) atmosphere.

${ }^{\mathrm{b}}$ DTBP (98\%, Sigma-Aldrich. All other experiments were conducted with DTBP (91\%, Polifin, now Sasol Polymers). More detailed analyses of the DTBP are given in the Supporting Information (Tables 1 and 2).

${ }^{\mathrm{c}}$ Experiments 1,2, 6-17 and 39-43 were conducted with 1-octene (98\%, Sigma-Aldrich, by analysis 99.3\%) as feed. All other experiments were conducted with pentene (Sasol Synfuels, Secunda) as feed. The feed compositions, as analysed by GC, are listed in Table 1 (octene) and Table 2 (pentene). A more detailed composition of the pentene is given in the supporting information (Table 3).

${ }^{\mathrm{d}}$ All material heavier than the feed was considered product. Only the liquid products were analysed by GC.

${ }^{\mathrm{e}}$ Experiment was conducted with a glass liner in the autoclave.

${ }^{\mathrm{f}}$ Ethanol added.

${ }^{\mathrm{g}}$ Experiments conducted at a pressure of $20 \mathrm{bar}_{\mathrm{g}}$. All other experiments were conducted at a pressure of $10 \mathrm{bar}_{\mathrm{g}}$.

${ }^{\text {h }}$ Benzene (99\%, NT Laboratory Supplies) 